\title{
EXISTENCE OF OPTIMAL CONTROLS FOR SYSTEMS GOVERNED BY SECOND ORDER LINEAR PARABOLIC PARTIAL DELAY-DIFFERENTIAL EQUATIONS WITH FIRST BOUNDARY CONDITIONS
}

\author{
K. L. TEO \\ (Received 20 April 1978) \\ (Revised 9 October 1978)
}

\begin{abstract}
In this paper, we consider a class of systems governed by second order linear parabolic delay-partial differential equations with first boundary conditions. Our main results are reported in Theorems 3.1 and 3.2. As in [9, Theorems 4.1 and 4.2], the coefficients and forcing terms of the system considered in Theorem 3.1 are linear in the control variables. On the other hand, the forcing terms of the system considered in Theorem 3.2 are allowed to be nonlinear in the control variables at the expense of dropping the control variables in the cost integrand.
\end{abstract}

\section{Introduction}

It appears that there are only few results $[5$, p. $262 ; 9,11]$ available in the literature on the existence of optimal controls for systems governed by parabolic partial delay-differential equations with controls and delayed arguments appearing in the coefficient.

In this paper, we present two existence theorems for optimal controls (Theorems 3.1 and 3.2). As in [9, Theorems 4.1 and 4.2], the coefficients and forcing terms of the system considered in Theorem 3.1 are linear in the control variables. However, Theorem 3.1 contains Theorems 4.1 and 4.2 of [9] as special cases. On the other hand, the coefficients and forcing terms of the system considered in Theorem 3.2 are allowed to be nonlinear in the control variables at the expense of dropping the control variables in the cost integrand. 
It is known [10] that a class of stochastic optimal control problems can be converted into a class of optimal control problems of systems governed by parabolic partial differential equations. This reduced problem is contained as a very special case of the class of optimal control problems currently under consideration.

Let $\Omega$ be a domain in $n$-dimensional Euclidean space $R^{n}$, bounded by a smooth surface $\partial \Omega$ satisfying the following properties: each point of $\partial \Omega$ is locally representable by functions with the Hölder continuous second order partiai derivatives.

We denote the coordinates of a point $x$ in $R^{n}$ by $x_{1}, \ldots, x_{n}$, time by $t$. Let $T$ be a fixed time instant, $T<\infty$, and $h_{\kappa}(\kappa=0,1, \ldots, \nu)$ be certain given numbers such that $0=h_{0}<h_{1}<\ldots<h_{\nu}<T$. We denote the intervals $I_{0}=\left[-h_{\nu}, 0\right], I_{1}=(0, T)$, $I_{2}=\left[-h_{\nu}, T\right]$.

Now let us consider the following second order linear partial delayed differential equations of parabolic type with a first boundary condition.

$$
\begin{aligned}
& \phi_{l}(u)(x, t)=\sum_{i, j=1}^{n} a_{i j}(x, t) \cdot \phi_{x_{i} x_{j}}(u)(x, t) \\
& +\sum_{\kappa=0}^{\nu} \sum_{i=1}^{n} b_{i, \kappa}\left(x, t-h_{\kappa}, u\left(x, t-h_{\kappa}\right)\right) \cdot \phi_{x_{i}}(u)\left(x, t-h_{\kappa}\right) \\
& +\sum_{\kappa=0}^{\nu} c_{\kappa}\left(x, t-h_{\kappa}, u\left(x, t-h_{\kappa}\right)\right) \cdot \phi(u)\left(x, t-h_{\kappa}\right) \\
& +\sum_{\kappa=0}^{\nu} f_{\kappa}\left(x, t-h_{k}, u\left(x, t-h_{\kappa}\right)\right), \quad \text { for }(x, t) \in \Omega \times I_{1} \\
& \phi(u)(x, t)=\phi_{0}(x, t), \\
& \text { for }(x, t) \in \Omega \times I_{0} \\
& \phi(u)(x, t)=0, \\
& \text { for }(x, t) \in \partial \Omega \times I_{2} \\
& u \in D,
\end{aligned}
$$

where $\psi_{t}=\partial \psi / \partial t, \psi_{x_{i}}=\partial \psi / \partial x_{i}, \psi_{x_{i} x_{j}}=\partial^{2} \psi / \partial x_{i} \partial x_{j}(i, j=1, \ldots, n)$, and $D$ is the set of admissible controls to be defined later.

Let $\hat{u}$ be a bounded measurable function from $\bar{\Omega} \times\left[-h_{\nu}, 0\right)$ into $R^{r}$ and let $U$ be a non-empty compact convex subset of $R^{r}$. Now let us define the set of admissible controls on $\bar{\Omega} \times\left[-h_{\nu}, T\right]$ by

$$
\begin{aligned}
D=\{u: & u \text { measurable on } \bar{\Omega} \times\left[-h_{\nu}, T\right], u(x, t)=\hat{u}(x, t) \\
& \text { almost everywhere on } \bar{\Omega} \times\left[-h_{\nu}, 0\right) \text { and } u(x, t) \in U \\
& \text { almost everywhere on } \bar{\Omega} \times[0, T]\} .
\end{aligned}
$$




\section{Auxiliary results}

With reference to system (1), it is assumed that, for each $u \in D$, the coefficients, forcing terms and data are defined and measurable on their appropriate domains. Before stating more specific assumptions, we shall introduce some useful notations.

Let $|0|$ denote the Lebesgue measure of the measurable set 0 of any finite dimensional Euclidean space. Let $E$ be any connected subset of an $s$-dimensional Euclidean space $R^{s}$ and denote by $C^{\prime}(E)$ the class of all $l$ times continuously differentiable functions on $E$, where $1 \leqslant l<\infty$ is an integer. Further, let $C_{0}^{l}(E)$ be the class of functions from $C^{\prime}(E)$ with compact support on $E$. For any $Z \in R^{s}$, let $|Z|=\left(\sum_{i=1}^{s} Z_{i}^{2}\right)^{\ddagger}$. Let $z_{x}=\left[z_{x_{i}}, \ldots, z_{x_{n}}\right]$ denote the gradient of the scalar valued function on $R^{n}$.

Let $E$ be as before and denote by $L^{\delta}(E), \delta \geqslant 1$, the Banach space consisting of all measurable functions on $E$ that are $\delta t h$-power integrable on $E$. The norm on it is defined by the equalities

$$
\|z\|_{\delta, E}=\left\{\int_{E}|z(y)|^{\delta} d y\right\}^{1 / \delta} \text { for } 1 \leqslant \delta<\infty
$$

and

$$
\|z\|_{\infty, E}=\underset{E}{\operatorname{ess} \sup }|z(y)| \quad \text { for } \delta=\infty .
$$

Measurability and integrability are to be understood in the sense of Lebesgue. The elements of $L^{\delta}(E)$ are the equivalence classes of the functions on $E$ (functions belonging to the same equivalence class are equal almost everywhere).

Let $\lambda$ be a real number such that $1 \leqslant \lambda<\infty$ and denote by $W_{\lambda}^{2,1}(E)$ the Banach space of functions from $L^{\lambda}(E)$ having generalized derivatives of the form $\left(\partial^{r} / \partial t^{r}\right)\left(\partial^{s} / \partial x^{s}\right)$ with any $r$ and $s$ satisfying the inequality $2 r+s \leqslant 2$. The norm on it is defined by the equality

$$
\|z\|_{\lambda, E}^{(2)}=\|z\|_{\lambda, E}+\left\|\frac{\partial z}{\partial t}\right\|_{\lambda, E}+\sum_{i=1}^{n}\left\|\frac{\partial z}{\partial x_{i}}\right\|_{\lambda, E}+\sum_{i, j=1}^{n}\left\|\frac{\partial^{2} z}{\partial x_{i} \partial x_{j}}\right\|_{\lambda, E}
$$

Note that if $z$ is only a function of $x$ defined in $\Omega$ then we denote $W_{\lambda}^{2}(\Omega)$ the Banach space of functions from $L^{\lambda}(\Omega)$ having generalized derivatives of the form $\partial^{8} / \partial x^{8}$ with $s=0,1$ and 2 . The norm on it is defined by the equality

$$
\|z\|_{\lambda, \Omega}^{(2)}=\|z\|_{\lambda, \Omega}+\sum_{i=1}^{n}\left\|\frac{\partial z}{\partial x_{i}}\right\|_{\lambda, \Omega}+\sum_{i, j=1}^{n}\left\|\frac{\partial^{2} z}{\partial x_{i} \partial x_{j}}\right\|_{\lambda, \Omega} .
$$

For any non-integral positive number $\lambda, H^{\lambda, \lambda / 2}(E)$ denotes the Banach space of functions $z$ that are continuous on $E$ and have derivatives of the form

$$
D_{i}^{\alpha} \cdot D_{x}^{\beta} \cdot z=\frac{\partial^{\alpha}}{\partial t^{\alpha}} \cdot\left(\frac{\partial \beta_{1}+\ldots+\beta_{n}}{\partial x_{1}^{\beta_{1}} \ldots \partial x_{n}^{\beta} n} \cdot z\right), \quad \sum_{i=1}^{n} \beta_{i}=\beta,
$$


(each $\beta_{i}$ is a non-negative integer, $2 \alpha+\beta<\lambda$ ), and have a finite norm

$$
|z|_{\bar{E}}^{\lambda}=\|z\|_{\bar{E}^{(\lambda)}}+\sum_{j=0}^{[\lambda]}\|z\|_{\bar{E}}^{(j)} .
$$

Note that $[\lambda]$ denotes the largest integral part of $\lambda$ and

$$
\begin{aligned}
& \|z\|_{\bar{E}^{(0)}}=|z|_{\bar{E}^{(0)}}=\max _{\bar{E}}|z(x, t)|, \\
& \|z\|_{\bar{E}^{(j)}}=\sum_{2 \alpha+\beta=j}\left|D_{l}^{\alpha} \cdot D_{x}^{\beta} \cdot z\right|_{\bar{E}}^{(0)},
\end{aligned}
$$

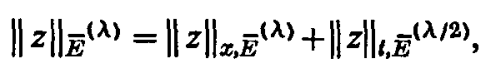

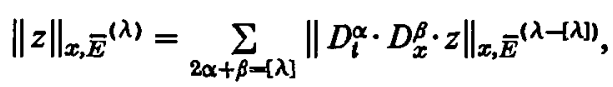

$$
\begin{aligned}
& \|z\|_{l, \bar{E}^{(\lambda / 2)}}=\sum_{0<\lambda-2 \alpha-\beta<2}\left\|D_{l}^{\alpha \cdot} \cdot D_{x}^{\beta} \cdot z\right\|_{l, \bar{E}} \overline{\bar{E}}^{\ddagger(\lambda-2 \alpha-\beta)}, \\
& \|z\|_{x \cdot \bar{E}^{(\gamma)}}=\max _{(x, t),\left(x^{\prime},\right) \in \bar{E}} \frac{\left|z(x, t)-z\left(x^{\prime}, t\right)\right|}{\left|x-x^{\prime}\right| \gamma}, \quad 0<\gamma<1 \text {, } \\
& \|z\|_{t, \bar{E}}(\gamma)=\max _{(x, t),\left(x, t^{\prime}\right) \in \bar{E}} \frac{\left|z(x, t)-z\left(x, t^{\prime}\right)\right|}{\left|t-t^{\prime}\right|^{\gamma}}, 0<\gamma<1 .
\end{aligned}
$$

Throughout this paper, the coefficients, the forcing terms and data of the system (1) are assumed to satisfy the following assumptions which will be referred to collectively as $(A)$.

(i) $a_{i j}(\cdot, \cdot)(i, j=1, \ldots, n)$ are continuous on $\bar{Q}$, where $Q=\Omega \times I_{1}$ and $\bar{Q}$ is the closure of $Q$,

(ii) there exist numbers $\alpha_{u}>\alpha_{l}>0$ such that

$$
\alpha_{u}|Z|^{2} \geqslant \sum_{i, j=1}^{n} a_{i j}(x, t) \cdot Z_{i} \cdot Z_{j} \geqslant \alpha_{l} \cdot|Z|^{2}
$$

for all $Z \in R^{n}$ uniformly on $\bar{Q}$ (uniformly parabolic), where $|Z|^{2}=\sum_{i=1}^{n}\left|Z_{i}\right|^{2}$,

(iii) $\max _{1 \leqslant i, j \leqslant n} \frac{\left|a_{i j}(x, t)-a_{i j}\left(x^{\prime}, t^{\prime}\right)\right|}{\left|t-t^{\prime}\right|+\left|x-x^{\prime}\right|} \leqslant M$,

where $t, t^{\prime} \in[0, T] ; x, x^{\prime} \in \bar{\Omega}$; and $M$ is a constant,

(iv) $b_{i, \kappa}\left(\cdot, \cdot-h_{\kappa}, \cdot\right), c_{\kappa}\left(\cdot, \cdot-h_{\kappa}, \cdot\right), f_{\kappa}\left(\cdot, \cdot-h_{\kappa}, \cdot\right)(i=1, \ldots, n ; \kappa=0,1, \ldots, v)$ are bounded measurable on $\bar{Q} \times U$ and continuous on $U$ for almost all $(x, t) \in \bar{Q}$,

(v) $\phi_{0} \in C^{2}\left(\bar{\Omega} \times\left[-h_{\nu}, 0\right]\right)$ and $\phi_{0}(x, t)=0$ for all $x \notin \bar{\Omega}_{0}$ and $t \in\left[-h_{\nu}, 0\right]$, where $\Omega_{0}$ is a compact subset of $\Omega$.

Note that all the results to be presented in this paper remain valid if the condition $\phi_{0} \in C^{2}\left(\Omega \times\left[-h_{\nu}, 0\right]\right)$ is replaced by a weaker condition $\phi_{0} \in W_{\lambda}^{2,1}\left(\bar{\Omega} \times\left[-h_{\nu}, 0\right]\right)$. 
The reason for imposing this more restrictive assumption is merely for convenience.

With this preparation, we state the following optimal control problem $P$.

Given the dynamic system (1), find a control $u_{0} \in D$ that minimizes the cost functional

$$
J(u)=\int_{Q} \int G\left(x, t, u(x, t), \phi(u)(x, t), \phi_{x}(u)(x, t)\right) d x d t,
$$

where

$$
\begin{aligned}
& G\left(x, t, u(x, t), \phi(u)(x, t), \phi_{x}(u)(x, t)\right) \\
& \quad=G_{1}(x, t, u(x, t))+G_{2}\left(x, t, \phi(u)(x, t), \phi_{x}(u)(x, t)\right) .
\end{aligned}
$$

For brevity, the following assumptions on the cost integrand $G$ will be referred to collectively as $\mathscr{G}$.

(i) $G_{1}(x, t, v)$ is measurable in $\bar{Q}$ for each $v \in R^{r}$, continuous in $R^{r}$ for each $(x, t) \in \bar{Q}$ and convex in $U$ for each $(x, t) \in \bar{Q}$; further, there exists a nonnegative function $g_{1} \in L^{1}\left(Q, R^{1}\right)$ and a constant $\omega>0$ so that

$$
\left|G_{1}(x, t, v)\right| \leqslant g_{1}(x, t)+\omega|v|^{\sigma}
$$

for some constant $\sigma \geqslant 1$ almost everywhere on $Q$; and

(ii) there exist a real number $\gamma \geqslant 0$ and a non-negative measurable function $g_{2} \in L^{1}\left(Q, R^{1}\right)$ so that

$$
\left|G_{2}\left(x, t, \psi^{0}, \psi^{1}, \ldots, \psi^{n}\right)\right| \leqslant g_{2}(x, t)+\gamma \sum_{\rho=0}^{n}\left|\psi^{\rho}\right|
$$

almost everywhere on $Q$.

For ease in future references, the statement: " $C$ depends on the structure of the differential equation of system (1)" will be used to mean that $C$ is determined by the quantities $\alpha_{l}, \alpha_{u}, M$, and the bounds of the functions $b_{i, k}, c_{\kappa}(i=1, \ldots, n$; $\kappa=0,1, \ldots, n)$.

Corresponding to system (1) we need the following definition.

Definition 2.1. A function $\phi: \bar{\Omega} \times I_{2} \rightarrow R^{1}$ is said to be a solution of system (1) if

(i) $\phi(x, t)=\phi_{0}(x, t)$ on $\Omega \times I_{0}$;

(ii) $\phi(x, t)=0$ on $\partial \Omega \times I_{2}$;

(iii) the restriction of $\phi$ to $Q$ belongs to $W_{\lambda}^{2,1}(Q)(n+2<\lambda<\infty)$; and

(iv) $\phi$ satisfies the differential equation of system (1) almost everywhere in $Q$.

Note that, in Definition 2.1, $\phi_{t}$ and $\phi_{x_{i} x_{j}}$ are, respectively, understood to be the generalized derivative of $\phi$ with respect to $t$ and the generalized derivative of $\phi_{x_{t}}$ with respect to $x_{j}$. 
The existence and uniqueness of solutions of system (1) together with two a priori estimates are known [8, Theorem 2.3, pp. 123-124]. This result is quoted without proof in the following theorem.

THEOREM 2.1. Under the assumptions (A), system (1) admits, for each $u \in D, a$ unique solution $\phi(u)$. Further $\phi(u)$ satisfies the following estimates.

and

$$
\|\phi(u)\|_{\lambda, e^{(2)} \leqslant N_{1}}
$$

$$
|\phi(u)|_{\bar{Q}}^{2+\mu} \leqslant N_{2}
$$

for all $\lambda>n+2$ and $\mu=1-((n+2) / \lambda)$, where the positive constants $N_{1}$ and $N_{2}$ depend only on $n, \lambda, \nu, Q, \partial \Omega$, the structure of the differential equation of system (1), the bounds for the functions $\left.f_{\kappa}(\kappa=0,1, \ldots, \nu),\left|\phi_{0}\right|_{\bar{\Omega} \times\left[-h_{v}, 0\right]}^{(0)},\left|\partial \phi_{0} / \partial x_{i}\right|_{\bar{\Omega} \times\left[-h_{\nu}, 0\right]}\right]^{(0)}(i=1, \ldots, n)$ and $\left\|\phi_{0}(\cdot, 0)\right\|_{\lambda, \Omega}{ }^{(2)}$.

In the sequel, Theorem 3.2 of [9] will be quoted without proof in the following theorem.

THEOREM 2.2. Let $\left\{u_{l}\right\}_{l=1}^{\infty} \subset D$ and let $\left\{\phi\left(u_{l}\right)\right\}_{l=1}^{\infty}$ be the corresponding sequence of solutions of system (1). Suppose that the assumptions $(A)$ are satisfied. Then there exists a subsequence $\left\{u_{l_{b}}\right\}_{l=1}^{\infty} \subset\left\{u_{l}\right\}_{l=1}^{\infty}$ so that

$$
\begin{aligned}
b_{i, \kappa}\left(\cdot, \cdot-h_{\kappa}, u_{l_{l}}(\cdot, \cdot-h)\right), c_{\kappa}\left(\cdot, \cdot-h_{\kappa}, u_{l_{\imath}}(\cdot, \cdot-\kappa)\right), \\
\quad f_{\kappa}\left(\cdot, \cdot-h_{\kappa}, u_{l}\left(\cdot, \cdot-h_{\kappa}\right)\right)(i=1, \ldots, n ; \kappa=0,1, \ldots, \nu)
\end{aligned}
$$

converge, respectively, to

$$
b_{i \kappa}^{*}\left(\cdot, \cdot-h_{\kappa}\right), e_{\kappa}^{*}\left(\cdot, \cdot-h_{k}\right), f_{\kappa}^{*}\left(\cdot, \cdot-h_{\kappa}\right)(i=1, \ldots, n ; \kappa=0,1, \ldots, \nu)
$$

in the weak ${ }^{*}$ topology of $L^{\infty}\left(Q, R^{1}\right)$ as $\imath \rightarrow \infty$. Further,

$$
\begin{aligned}
& \left.\begin{array}{rl}
\phi\left(u_{l_{i}}\right) & \rightarrow \phi^{*} \\
\phi_{x_{i}}\left(u_{l_{l}}\right) \rightarrow \phi_{x_{i}}^{*}, \quad i=1, \ldots, n,
\end{array}\right\} \text { uniformly on } \bar{Q} \\
& \left.\begin{array}{c}
\phi_{l}\left(u_{k}\right) \rightarrow \phi_{l}^{*} \\
\phi_{x_{i} x_{j}}\left(u_{l_{l}}\right) \rightarrow \phi_{x_{1} x_{j}}^{*}, \quad i, j=1, \ldots, n,
\end{array}\right\} \text { weakly in } L^{\lambda}(Q) \text { as } \iota \rightarrow \infty \text {, }
\end{aligned}
$$


where $\lambda>n+2$ and $\phi^{*}$ is the unique solution of the system

$$
\begin{aligned}
& \phi_{l}^{*}(x, t)=\sum_{i, j=1}^{n} a_{i j}(x, t) \phi_{x_{i} x_{j}}^{*}(x, t) \\
& +\sum_{\kappa=0}^{p} \sum_{i=1}^{n} b_{i, \kappa}^{*}\left(x, t-h_{\kappa}\right) \cdot \phi_{x_{i}}^{*}\left(x, t-h_{\kappa}\right) \\
& +\sum_{\kappa=0}^{\nu} c_{\kappa}^{*}\left(x, t-h_{\kappa}\right) \cdot \phi^{*}\left(x, t-h_{\kappa}\right) \\
& +\sum_{\kappa=0}^{\nu} f_{\kappa}^{*}\left(x, t-h_{\kappa}\right), \quad(x, t) \in Q, \\
& \phi^{*}(x, t)=\phi_{0}(x, t) \\
& (x, t) \in \Omega \times I_{0}, \\
& \phi^{*}(x, t)=0, \\
& \left.(x, t) \in \partial \Omega \times I_{2} .\right)
\end{aligned}
$$

For brevity, let us introduce the following notation.

$$
F_{\kappa}(x, t, v)=\left[b_{1, \kappa}(x, t, v), b_{2, \kappa}(x, t, v), \ldots, b_{n, \kappa}(x, t, v), c_{\kappa}(x, t, v), f_{\kappa}(x, t, v)\right]
$$

$(\kappa=0,1, \ldots, v)$;

$(\kappa=0,1, \ldots, v)$; and

$$
\hat{F}_{\kappa}(x, t, v)= \begin{cases}F_{\kappa}(x, t, v), & (x, t) \in \Omega \times\left[0, T-h_{\kappa}\right], \\ F_{\kappa}\left(x, T-h_{\kappa}, v\right) & (x, t) \in \Omega \times\left(T-h_{\kappa}, T\right]\end{cases}
$$

$$
\tilde{F}(x, t)=\left\{\left[\hat{F}_{0}(x, t, v), \hat{F}_{1}(x, t, v), \ldots, \hat{F}_{\nu}(x, t, v)\right]^{\mathrm{T}}: v \in U\right\},
$$

where the superscript " $T$ " denotes transpose.

Clearly, $\widetilde{F}$ is a set-valued function from $\bar{Q}$ into $R^{(n+2)(\nu+1)}$.

In the sequel, we need

Definition 2.2. A set-valued function $\tilde{F}: \bar{\Omega} \times[0, T] \rightarrow R^{(n+2)(\nu+1)}$ is said to be measurable if $\{(x, t) \in \bar{\Omega} \times[0, T]: B \cap \tilde{F}(x, t) \neq \varnothing\}$ is measurable for every closed subset $B$ of $R^{(n+2)(\nu+1)}$.

As an immediate consequence of [3, Theorem 3, p. 281], we have the following Filippov-type lemma.

LEMMA 2.1. Let $b_{i, \kappa}, c_{\kappa}, f_{\kappa}(i=1, \ldots, n ; \kappa=0,1, \ldots, \nu)$ satisfy the condition (iv) of the assumptions $(A)$ and let $z$ be a measurable function from $\bar{Q}$ into $R^{(n+2)(v+1)}$ so that $z(x, t) \in \widetilde{F}(x, t)$ a.e. on $\bar{Q}$. If $\widetilde{F}$ is a measurable set-valued function, then, there exists a measurable function $u^{*}$ on $\bar{Q}$ such that

$$
z(x, t)=\left[\hat{F}_{0}\left(x, t, u^{*}(x, t)\right), \hat{F}_{1}\left(x, t, u^{*}(x, t)\right), \ldots, \hat{F}_{\nu}\left(x, t, u^{*}(x, t)\right)\right]^{\mathrm{T}}
$$

and $u^{*}(x, t) \in U$ a.e. on $\bar{Q}$. 
Using Theorem 2.2 and Lemma 2.1, we obtain

LEMMA 2.2. Let the assumptions of Theorem 2.2 be satisfied and let $\widetilde{F}(x, t)$ be convex for each $(x, t) \in \bar{Q}$. If, in addition, $\tilde{F}$ is a measurable set-valued function, there exists a control $u^{*} \in D$ so that

$$
\begin{gathered}
b_{i, k}^{*}\left(x, t-h_{k}\right)=b_{i, k}\left(x, t-h_{\kappa}, u^{*}\left(x, t-h_{\kappa}\right)\right), \\
c_{\kappa}^{*}\left(x, t-h_{\kappa}\right)=c_{\kappa}\left(x, t-h_{\kappa}, u^{*}\left(x, t-h_{\kappa}\right)\right), \\
f_{k}^{*}\left(x, t-h_{\kappa}\right)=f_{\kappa}\left(x, t-h_{\kappa}, u^{*}\left(x, t-h_{\kappa}\right)\right)
\end{gathered}
$$

$(i=1, \ldots, n ; \kappa=0,1, \ldots, \nu)$ almost everywhere on $\bar{Q}$.

PROOF. Let $\left\{u_{l}\right\}_{l=1}^{\infty} \subset\left\{u_{l_{l}}^{\infty} \subset D\right.$ be as defined in Theorem 2.2. Let

$$
H^{\prime}(x, t)=\left[\hat{F}_{0}\left(x, t, u_{h^{\prime}}(x, t)\right), \hat{F}_{1}\left(x, t, u_{l_{i}}(x, t)\right), \ldots, \hat{F}_{\nu}\left(x, t, u_{l_{i}}(x, t)\right)\right]^{\mathrm{T}}
$$

and let the set $\mathscr{N}$ be defined by

$$
\mathscr{N}=\{y: y \text { measurable on } \bar{Q} \text { and } y(x, t) \in \tilde{F}(x, t) \text { for almost all }(x, t) \in \bar{Q}\} .
$$

Recall that $U$ is non-empty. Thus, $D$ is non-empty. Now, by using appropriate assumptions of $(A)$, we see that $\left[\hat{F}_{0}(\cdot, \cdot, u(\cdot, \cdot)), \hat{F}_{1}(\cdot, \cdot, u(\cdot, \cdot)), \ldots, \hat{F}_{\nu}(\cdot, \cdot, u(\cdot, \cdot))\right]^{\mathrm{T}}$ is measurable on $G$ for any $u \in D$. Further, by the definition of $\tilde{F}$, it is clear that $\left[\hat{F}_{0}(x, t, u(x, t)), \hat{F}_{1}(x, t, u(x, t)), \ldots, \hat{F}_{\nu}(x, t, u(x, t))\right]^{\mathrm{T}} \in \tilde{F}(x, t)$ for all $(x, t) \in G$ for any $u \in D$. Thus, the set $\mathscr{N}$ is non-empty.

By hypothesis, $\widetilde{F}(x, t)$ is convex for each $(x, t) \in \bar{Q}$. Thus, it is easily observed that $\mathscr{N}$ is convex. On the other hand, it follows from the definitions of $\widetilde{F}$ (equation 8 ) and $\mathscr{N}$ and the condition (iv) of the assumptions $(A)$ that, for each $(x, t) \in \bar{Q}$, $\widetilde{F}(x, t)$ is a compact subset of $R^{(n+2)(\nu+1)}$ and there exists a constant $K>0$ so that

$$
|y(x, t)| \leqslant K
$$

almost everywhere in $\bar{Q}$ for all $y \in \mathscr{N}$. Let $\tilde{\mathscr{N}}$ be the class of functions defined by

$$
\begin{gathered}
\tilde{N}=\left\{z: z \text { measurable on } \bar{Q} \text { with values in } R^{(n+2)(\nu+1)}\right. \\
\text { and }|z(x, t)| \leqslant K \text { for almost all }(x, t) \in \bar{Q}\} .
\end{gathered}
$$

Clearly, $\mathscr{N} \subset \tilde{\mathscr{N}}$. From [2, Theorem 7.1, p. 19], the closed unit ball of $L^{\infty}\left(Q, R^{(n+2)(\nu+1)}\right)$ is compact in the weak * topology of $L^{\infty}\left(Q, R^{(n+2)(\nu+1)}\right)$; clearly a closed ball of any finite radius is also compact in the weak * topology of $L^{\infty}\left(Q, R^{(n+2)(\nu+1)}\right.$. This obviously implies that $\tilde{\mathscr{N}}$ is compact in the weak * topology of $L^{\infty}\left(Q, R^{(n+2)(v+1)}\right)$. Now, in view of the definitions of the $\hat{F}_{\kappa}(\kappa=0,1, \ldots, \nu)$ and $H^{\prime}$, we observe that $\left\{H^{\prime}\right\}_{l=1}^{\infty} \subset \mathscr{N} \subset \tilde{N}$. On the other hand, it follows from Theorem 
2.2 and the definitions of the $\hat{F}_{\kappa}(\kappa=0,1, \ldots, \nu)$ that

$$
\hat{F}_{\kappa}\left(\cdot, \cdot, u_{l}(\cdot, \cdot)\right) \rightarrow \hat{F}_{\kappa}^{*}(\cdot, \cdot) \quad(\kappa=0,1, \ldots, v)
$$

in the weak * topology of $L^{\infty}\left(Q, R^{n+2}\right)$ as $\iota \rightarrow \infty$, where

and

$$
F_{\kappa}^{*}(x, t)= \begin{cases}F_{k}^{*}(x, t), & (x, t) \in \Omega \times\left[0, T-h_{\kappa}\right], \\ F_{\kappa}^{*}\left(x, T-h_{\kappa}\right), & (x, t) \in \Omega \times\left(T-h_{\kappa}, T\right]\end{cases}
$$

$$
F_{\kappa}^{*}(x, t)=\left[b_{1, \kappa}^{*}(x, t), \ldots, b_{n, \kappa}^{*}(x, t), c_{\kappa}^{*}(x, t), f_{\kappa}^{*}(x, t)\right] \quad(\kappa=0,1, \ldots, \nu) .
$$

This, in turn, implies that $H^{\prime}(\cdot, \cdot) \rightarrow H^{*}(\cdot, \cdot)$ in the weak * topology of $L^{\infty}\left(Q, R^{(n+2)(\nu+1)}\right)$, where

$$
H^{*}(x, t)=\left[\hat{F}_{0}^{*}(x, t), \hat{F}_{1}^{*}(x, t), \ldots, \hat{F}_{\nu}^{*}(x, t)\right]^{\mathrm{T}} .
$$

Since $\left\{H^{t}\right\}_{i=1}^{\infty} \subset \mathscr{N} \subset \tilde{N}$ and $\tilde{\mathscr{N}}$ is compact in the weak * topology of $L^{\infty}\left(Q, R^{(n+2)(\nu+1)}\right)$, it follows that $H^{*} \in \tilde{\mathscr{N}}$. Next, we shall show that $H^{*} \in \mathscr{N}$. This is equivalent to showing that $H^{*}(x, t) \in \tilde{F}(x, t)$ almost everywhere on $\bar{Q}$. For each $\iota=1,2, \ldots$, let

$$
Q_{\iota}=\left\{(x, t) \in \bar{Q}: u_{\iota}(x, t) \notin U\right\}
$$

Let $\tilde{Q}=\bigcup_{i=1}^{\infty} Q_{t}$. Since $u_{l_{l}}(x, t) \in U$ almost everywhere on $\bar{Q}$ for all integers $\imath \geqslant 1$, it follows that $|\tilde{Q}|=0$. By hypothesis, $\tilde{F}$ is a measurable set-valued function defined on $\bar{Q}$. Thus, by virtue of Theorem 1 of $[6, \mathrm{p}$. 857], there exists, for any $\varepsilon_{1}>0$, an open set $E_{1} \subset \bar{Q} \backslash \widetilde{Q}=Q^{1}$ so that $\left|E_{1}\right|<\varepsilon_{1}$ and $\widetilde{F}(\cdot, \cdot)$ is continuous in the Hausdorff metric topology on $Q^{\mathbf{1}} \backslash E_{1}$. Now, by Lusin's Theorem, there exists, for any $\varepsilon_{2}>0$, an open set $E_{2} \subset Q^{1}$ so that $\left|E_{2}\right|<\varepsilon_{2}$ and $H^{*}(\cdot, \cdot)$ is continuous on $Q^{1} \backslash E_{2}$. Let $E=E_{1} \cup E_{2}$. Then, it is obvious that $|E|<\varepsilon_{1}+\varepsilon_{2}=\varepsilon_{3}$. Further, $\tilde{F}(\cdot, \cdot)$ is continuous in the Hausdorff metric topology on $Q^{1} \backslash E=Q^{2}$ and $H^{*}(\cdot, \cdot)$ is continuous on $Q^{2}$. Let $\left(x_{0}, t_{0}\right) \in Q^{2}$ be arbitrary but fixed. Then, for any $\varepsilon_{4}>0$, there exists a $\delta=\delta\left(\varepsilon_{4}\right)$ such that

$$
\tilde{F}(x, t) \subseteq \tilde{F}^{*}\left(x_{0}, t_{0}\right)
$$

whenever $\left|(x, t)-\left(x_{0}, t_{0}\right)\right|<\delta$, where $\widetilde{F}^{\star 4}(x, t)$ denotes the closed $\varepsilon_{4}$-neighborhood of $\tilde{F}(x, t)$. We shall now show that $H^{*}\left(x_{0}, t_{0}\right) \in \widetilde{F}\left(x_{0}, t_{0}\right)$. Since $\left(x_{0}, t_{0}\right)$ is a continuity point of the function $H^{*}$, it follows that, for any measurable set $\Delta \subset Q^{2}$ containing $\left(x_{0}, t_{0}\right)$ and contracting to the one point set $\left\{\left(x_{0}, t_{0}\right)\right\}$, we have

$$
H^{*}\left(x_{0}, t_{0}\right)=\lim _{|\Delta| \downarrow 0}\left\{\frac{1}{|\Delta|} \int_{\Delta} \int H^{*}(x, t) d x d t\right\} .
$$


Let $M_{\delta}=\left\{(x, t) \in Q^{2}:\left|(x, t)-\left(x_{0}, t_{0}\right)\right|<\delta\right\}$ and let $\mathscr{B}=\left\{B \in M_{\delta}: B\right.$ is measurable and contains $\left.\left(x_{0}, t_{0}\right)\right\}$.

Then, by virtue of the fact that $H\left(x_{0}, t_{0}, u_{l_{c}}\left(x_{0}, t_{0}\right)\right) \in \widetilde{F}\left(x_{0}, t_{0}\right)$ for all positive integers $\iota$, it follows from (9) that, for any $B \in \mathscr{B}$,

$$
H\left(x, t, u_{l_{c}}(x, t)\right) \in \tilde{F}^{e_{c}}\left(x_{0}, t_{0}\right)
$$

for all $(x, t) \in B$ and for all $\iota=1,2, \ldots$. Now, we note that $\tilde{F}^{e_{4}}\left(x_{0}, t_{0}\right)$ is convex and compact (and hence closed). Thus, it can be easily deduced from relation (11) that

$$
\frac{1}{|B|} \int_{B} \int H\left(x, t, u_{l_{l}}(x, t)\right) d x d t \in \tilde{F}^{\varepsilon 4}\left(x_{0}, t_{0}\right)
$$

for all $\iota=1,2, \ldots$ Further, since $\tilde{F}^{e_{4}}\left(x_{0}, t_{0}\right)$ is closed and $H\left(\cdot, \cdot, u_{l_{l}}(\cdot, \cdot)\right)$ converges to $H^{*}(\cdot, \cdot)$ in the weak * topology of $L^{\infty}\left(Q, R^{(n+2)(\nu+1)}\right)$, it follows from (12) that

$$
\lim _{t \rightarrow \infty} \frac{1}{|B|} \int_{B} \int H\left(x, t, u_{l_{\iota}}(x, t)\right) d x d t=\frac{1}{|B|} \int_{B} \int H^{*}(x, t) d x d t \in \tilde{F}^{\varepsilon_{4}}\left(x_{0}, t_{0}\right)
$$

for any $B \in \mathscr{B}$. Again, by the fact that $\tilde{F}^{s_{4}}\left(x_{0}, t_{0}\right)$ is closed, we deduce readily from (13) and (10) that if the set $B \in \mathscr{B}$ contracts to the one-point set $\left\{\left(x_{0}, t_{0}\right)\right\}$ then

$$
H^{*}\left(x_{0}, t_{0}\right)=\lim _{|B| \downarrow 0}\left\{\frac{1}{|B|} \int_{B} \int H^{*}(x, t) d x d t\right\} \in \tilde{F}^{e_{4}}\left(x_{0}, t_{0}\right) .
$$

Since $\varepsilon_{4}>0$ is arbitrary and $\tilde{F}\left(x_{0}, t_{0}\right)$ is closed, $H^{*}\left(x_{0}, t_{0}\right) \in \tilde{F}\left(x_{0}, t_{0}\right)$. Further, $\left(x_{0}, t_{0}\right) \in Q^{2}$ is also arbitrary. Thus, $H^{*}(x, t) \in \tilde{F}(x, t)$ for all $(x, t) \in Q^{2}$. Again, since $\varepsilon_{1}>0$ and $\varepsilon_{2}>0$ are arbitrary, $\varepsilon_{3}>0$ is arbitrary and hence it follows that $H^{*}(x, t) \in \tilde{F}(x, t)$ almost everywhere in $\bar{Q}$. At this stage, we can easily convince ourselves the validity of the lemma by using the definitions of the $\hat{F}_{\kappa}^{*}(\kappa=0,1, \ldots, \nu)$ $\mathscr{N}$ and Lemma 2.1.

Combining Theorem 2.2 and Lemma 2.2, we have

THEOREM 2.3. Let $\left\{u_{l}\right\}_{l=1}^{\infty} \subset D$ and let $\left\{\phi\left(u_{l}\right)\right\}_{l=1}^{\infty}$ be the corresponding set of solutions of the system (1). Let the set-valued function $\widetilde{F}$ defined by equation (8) be measurable and let $\tilde{F}(x, t)$ be convex for each $(x, t) \in \bar{Q}$. Suppose that the assumptions $(A)$ are satisfied. Then, there exist a subsequence $\left\{u_{i}\right\}_{l=1}^{\infty} \subset\left\{u_{l}\right\}_{l=1}^{\infty}$ and a control $u^{*} \in D$ so that

$$
\left.\begin{array}{c}
\phi\left(u_{l_{l}}\right) \rightarrow \phi\left(u^{*}\right) \\
\phi_{x_{i}}\left(u_{l_{l}}\right) \rightarrow \phi_{x_{i}}\left(u^{*}\right) \quad i=1, \ldots, n \\
\phi_{l}\left(u_{l_{l}}\right) \rightarrow \phi_{l}\left(u^{*}\right) \\
\phi_{x_{i} x_{i}}\left(u_{l_{l}}\right) \rightarrow \phi_{x_{i} x_{j}}\left(u^{*}\right), \quad i, j=1, \ldots, n
\end{array}\right\} \text { weakly in } L^{\lambda}(Q)
$$


as $\iota \rightarrow \infty$, where $\lambda>n+2$ and $\phi\left(u^{*}\right)$ is the unique solution of system (1) with $u$ replaced by $u^{*}$.

LEMMA 2.3. Let the function $Y$ satisfy the condition ( $i)$ of the assumptions $\mathscr{G}$. If $\left\{u_{l}\right\}_{l=1}^{\infty} \subset D$ and $u_{l}$ converges in the weak ${ }^{*}$ topology of $L^{\infty}\left(Q, R^{r}\right)$ to $u^{*} \in D$, then there exists a subsequence $\left\{u_{l_{l}}(\cdot, \cdot)\right\}_{l=1}^{\infty} \subset\left\{u_{l}(\cdot, \cdot)\right\}_{l=1}^{\infty}$ so that

$$
\lim _{t \rightarrow \infty} \int_{Q} \int Y\left(x, t, u_{l_{t}}(x, t)\right) d x d t \geqslant \int_{Q} \int Y\left(x, t, u^{*}(x, t)\right) d x d t
$$

Proof. By hypothesis, the sequence $\left\{u_{l}\right\}_{l=1}^{\infty} \subset D$ converges to $u^{*} \in D$ in the weak * topology of $L^{\infty}\left(Q, R^{r}\right)$. Since $|Q|<\infty$, the sequence also converges, in particular, weakly in $L^{1}\left(Q, R^{r}\right)$ to $u^{*}$. In view of the second part of the condition (i) of the assumptions $\mathscr{G}$ and the fact that $U$ is compact, we can easily show that the set defined by

$$
\{Y(\cdot, \cdot, u(\cdot, \cdot)): u \in D\}
$$

satisfies the sufficient conditions stated in [1, Corollary 11, p. 294] and hence it is compact in the weak topology of $L^{1}\left(Q, R^{r}\right)$. Thus, there exists a subsequence $\left\{Y\left(\cdot, \cdot, u_{l_{l}}(\cdot, \cdot)\right)\right\}_{l=1}^{\infty} \subset\left\{Y\left(\cdot, \cdot, u_{l}(\cdot, \cdot)\right)\right\}_{l=1}^{\infty}$ so that it converges to $Y^{*}(\cdot, \cdot)$ in the weak topology of $L^{1}\left(Q, R^{r}\right)$. Obviously, $\left\{u_{l_{l}}\right\}_{t=1}^{\infty} \subset\left\{u_{l}\right\}_{l=1}^{\infty}$ also converges weakly in $L^{1}\left(Q, R^{r}\right)$ to $u^{*}$. Thus, the sequence

$$
\left\{\left[\begin{array}{c}
u_{l_{l}}(\cdot, \cdot) \\
Y\left(\cdot, \cdot, u_{l_{l}}(\cdot, \cdot)\right)
\end{array}\right]\right\}_{l=1}^{\infty} \quad \text { converges to }\left[\begin{array}{l}
u^{*}(\cdot, \cdot) \\
Y^{*}(\cdot, \cdot)
\end{array}\right]
$$

weakly in $L^{1}\left(Q, R^{r+1}\right)$. By the Banach-Saks Theorem [7, p. 80], we can extract a subsequence

$$
\left\{\left[\begin{array}{c}
u_{l_{l}}^{k}(\cdot, \cdot) \\
Y\left(\cdot, \cdot, u_{l_{l}}^{\kappa}(\cdot, \cdot)\right)
\end{array}\right]\right\}_{k=1}^{\infty} \subset\left\{\left[\begin{array}{c}
u_{l_{l}}(\cdot, \cdot) \\
Y\left(\cdot, \cdot, u_{l_{l}}^{k}(\cdot, \cdot)\right)
\end{array}\right]\right]_{l=1}^{\infty}
$$

so that

$$
\frac{1}{\nu} \sum_{k=1}^{\nu}\left[\begin{array}{c}
u_{l_{l}}^{k}(\cdot, \cdot) \\
Y\left(\cdot, \cdot, u_{l_{l}}^{k}(\cdot, \cdot)\right)
\end{array}\right] \text { converges to }\left[\begin{array}{c}
u^{*}(\cdot, \cdot) \\
Y^{*}(\cdot, \cdot)
\end{array}\right]
$$

in the norm of $L^{1}\left(Q, R^{r+1}\right)$. This, in turn, implies that $(1 / v) \sum_{k=1}^{\nu} u_{l_{6}}^{k}(\cdot, \cdot)$ and $(1 / \nu) \sum_{k=1}^{\nu} Y\left(\cdot, \cdot u_{l_{h}}^{k}(\cdot, \cdot)\right)$ converge, respectively, to $u^{*}(\cdot, \cdot)$ in the norm of $L^{1}\left(Q, R^{r}\right)$ and $Y^{*}(\cdot, \cdot)$ in the norm of $L^{1}\left(Q, R^{1}\right)$ as $\nu \rightarrow \infty$. 
Now, by hypothesis, $Y(x, t, \cdot)$ is convex in $U$ for each $(x, t) \in \bar{Q}$, it follows that

$$
\begin{aligned}
\frac{1}{\nu} \sum_{k=1}^{\nu} \int_{Q} \int Y\left(x, t, u_{l_{l}}^{k}(x, t)\right) d x d t & =\int_{Q} \int \frac{1}{\nu} \sum_{k=1}^{\nu} Y\left(x, t, u_{l_{l}}^{k}(x, t)\right) d x d t \\
& \geqslant \int_{Q} \int Y\left(x, t, \frac{1}{\nu} \sum_{k=1}^{\nu} u_{l_{l}}^{k}(x, t)\right) d x d t
\end{aligned}
$$

For ease in references, the second part of the condition (i) of the assumptions $\mathscr{G}$ is recalled below. There exists some constant $\sigma \geqslant 1$ so that

$$
|Y(x, t, v)| \leqslant g_{1}(x, t)+\omega|v|^{\sigma}
$$

for almost all $(x, t) \in Q$ and for all $v \in R^{r}$.

Let us first consider the case when $\sigma=1$ (the case when $\sigma>1$ will be considered later). In this case, we obtain immediately from inequality (16) that

$$
\int_{Q} \int|Y(x, t, u(x, t))| d x d t \leqslant \int_{Q} \int g_{1}(x, t) d x d t+\omega \int_{Q}|u(x, t)| d x d t .
$$

Since $|Q|<\infty$ and $U$ is compact, it is clear that $Y$ is an operator mapping from $L^{1}\left(Q, R^{r}\right)$ into $L^{1}\left(Q, R^{1}\right)$. Thus, it follows from an obvious generalization of Theorem 2.1 of $\left[4\right.$, p. 22] that $Y$ is a continuous operator from $L^{1}\left(Q, R^{r}\right)$ into $L^{1}\left(Q, R^{1}\right)$. Since the sequence $\left\{(1 / v) \sum_{k=1}^{v} u_{l}^{k}(\cdot, \cdot)\right\}_{\nu=1}^{\infty}$ converges to $u^{*}$ in the norm of $L^{1}\left(Q, R^{r}\right)$ as $\nu \rightarrow \infty$, it follows that

$$
\lim _{\nu \rightarrow \infty} \int_{Q} \int Y\left(x, t, \frac{1}{\nu} \sum_{k=1}^{\nu} u_{l}^{k}(x, t)\right) d x d t=\int_{Q} \int Y\left(x, t, u^{*}(x, t)\right) d x d t .
$$

Next, we recall that the sequence $\left\{(1 / \nu) \sum_{k=1}^{v} Y\left(\cdot, \cdot, u_{l_{h}}^{k}(\cdot, \cdot)\right)\right\}_{\nu=1}^{\infty}$ converges to $Y^{*}(\cdot, \cdot)$ in the norm of $L^{1}\left(Q, R^{1}\right)$ as $\nu \rightarrow \infty$. Thus,

$$
\lim _{\nu \rightarrow \infty} \frac{1}{\nu} \sum_{k=1}^{\nu} \int_{Q} \int Y\left(x, t, u_{k_{t}^{k}}^{k}(x, t)\right) d x d t=\int_{Q} \int Y^{*}(x, t) d x d t .
$$

Further, since the sequence $\left\{Y\left(\cdot, \cdot, u_{\iota}(\cdot, \cdot)\right)\right\}_{c^{\infty}=1}^{\infty}$ converges to $Y^{*}(\cdot, \cdot)$ weakly in $L^{1}\left(Q, R^{1}\right)$ and $|Q|<\infty$, it is obvious that

$$
\lim _{t \rightarrow \infty} \int_{Q} \int Y\left(x, t, u_{\iota_{l}}(x, t)\right) d x d t=\int_{Q} \int Y^{*}(x, t) d x d t
$$

Thus, by combining (15), (19), (20) and (18), we obtain inequality (14) for the case when $\sigma=1$.

To complete the proof, we shall show that the lemma remains valid when $\sigma>1$. In fact, the proof is similar to that given for the case when $\sigma=1$ with only the following three minor modifications. 
(i) replace [1, Corollary 11, p. 294] by $[1$, Corollary 4 , p. 289];

(ii) replace $L^{1}\left(Q, R^{1}\right)$ and $L^{1}\left(Q, R^{r}\right)$ by $L^{\sigma}\left(Q, R^{1}\right)$ and $L^{\sigma}\left(Q, R^{r}\right)$ respectively everywhere; and

(iii) replace inequality (17) by the following inequality, which is obtained readily from inequality (16),

$$
\int_{Q} \int|Y(x, t, u(x, t))| d x d t \leqslant \int_{Q} \int g_{1}(x, t) d x d t+\omega \int_{Q} \int|u(x, t)|^{\sigma} d x d t .
$$

Thus, the proof is complete.

\section{Existence theorems for optimal controls}

In this section, we shall present two results on the existence of optimal controls for the problem $P$ in Theorems 3.1 and 3.2. The main tools for Theorem 3.1 are Theorem 2.2 and Lemma 2.3. On the other hand, Theorem 3.2 is proved on a basis of Theorem 2.3, while Theorem 2.3 was obtained by using Theorem 2.2 and Lemma 2.2. Note that, as in [9, Theorems 4.1 and 4.2], the coefficients and forcing terms of system (1) considered in Theorem 3.1 are assumed linear in the control variables. However, this result contains those reported in [9, Theorems 4.1 and 4.2] as special cases. In Theorem 3.2, the coefficients and forcing terms of system (1) are allowed to be non-linear in the control variables at the expense of dropping the control variables in the cost integrand.

THEOREM 3.1. Consider the problem P. Suppose that the assumptions $(A)$ and $G$ are satisfied. Further, it is assumed that the coefficients and forcing terms of the system (1) are linear in the control variables. Then the corresponding problem $P$ has a solution.

Proof. By the assumptions $\mathscr{G}$ and the fact that $U$ is compact and convex, it is easily deduced that $\operatorname{Inf}_{u \in D} J(u)>-\infty$. Clearly, there exists a sequence $\left\{u_{\}_{l}}^{\infty \infty} \subset D\right.$ so that

$$
\lim _{l \rightarrow \infty} J\left(u_{l}\right)=\operatorname{Inf}_{u \in D} J(u) .
$$

Since $D$ is a $w^{*}$-compact subset of $L^{\infty}\left(Q, R^{r}\right)$, there exists a subsequence of $\left\{u_{\}}\right\}_{l=1}^{\infty}$, which is denoted by the original sequence, so that it converges to $u^{*} \in D$ in the weak * topology of $L^{\infty}\left(Q, R^{r}\right)$. By hypothesis, the coefficients and forcing terms of the system (1) are linear in the control variables. Thus, it can be easily shown from Theorem 2.2 that $\phi\left(u_{i}\right)$ and $\phi_{x_{i}}\left(u_{i}\right)(i=1, \ldots, n)$ converge uniformly on $\bar{Q}$ to $\phi\left(u^{*}\right)$ and $\phi_{x_{i}}\left(u^{*}\right)(i=1, \ldots, n)$ respectively. 
Now, let $J_{1}$ denote the first component of $J$. Then, by virtue of the condition (i) of $\mathscr{G}$, it follows from Lemma 2.3 that we can extract a subsequence $\left\{u_{l}\right\}_{l=1}^{\infty} \subset\left\{u_{l}\right\}_{l=1}^{\infty}$ so that

$$
J_{1}\left(u^{*}\right) \leqslant \lim _{\imath \rightarrow \infty} J_{1}\left(u_{l_{l}}\right)
$$

On the other hand, we can easily verify from the condition (ii) of $\mathscr{G}$ and inequality (4) of Theorem 2.1 that the set defined by

$$
H=\left\{G_{2}\left(\cdot, \cdot, \phi(u)(\cdot, \cdot), \phi_{x}(u)(\cdot, \cdot)\right): u \in D\right\}
$$

satisfied the sufficient conditions stated in Corollary 11 of $[1$, p. 294]. Thus, it is compact in the weak topology of $L^{1}\left(Q, R^{1}\right)$. Next, by virtue of the condition (ii) of the assumptions $\mathscr{G}$, inequality (3) of Theorem 2.1 and the fact that $|Q|<\infty$, we can readily show that $G_{2}$ is an operator mapping from $L^{\lambda}\left(Q, R^{n+1}\right)$ into $L^{1}\left(Q, R^{1}\right)$. Thus, it follows from an obvious generalization of $\left[4\right.$, Theorem 2.1, p. 22] that $G_{2}$ is a continuous operator from $L^{\lambda}\left(Q, R^{n+1}\right)$ into $L^{1}\left(Q, R^{1}\right)$. Further, since $\phi\left(u_{l}\right)$ and $\phi_{x_{i}}\left(u_{i}\right)(i=1, \ldots, n)$ converge uniformly on $\bar{Q}$ to $\phi\left(u^{*}\right)$ and $\phi_{x_{i}}\left(u^{*}\right)(i=1, \ldots, n)$ res pectively and $|Q|<\infty$, it is clear that $\phi\left(u_{l_{l}}\right)$ and $\phi_{x_{i}}\left(u_{l_{l}}\right)(i=1, \ldots, n)$ converge, respectively, to $\phi\left(u^{*}\right)$ and $\phi_{x_{i}}\left(u^{*}\right)(i=1, \ldots, n)$ in the norm of $L^{\lambda}(Q)$ as $\iota \rightarrow \infty$, where $n+2<\lambda<\infty$. Thus,

$$
J_{2}\left(u_{l_{l}}\right)=\int_{Q} \int_{2} G_{2}\left(x, t, \phi\left(u_{l_{l}}\right)(x, t), \phi_{x}\left(u_{l_{l}}\right)(x, t)\right) d x d t
$$

converges to

$$
J_{2}\left(u^{*}\right)=\int_{Q} \int_{2}\left(x, t, \phi\left(u^{*}\right)(x, t), \phi_{x}\left(u^{*}\right)(x, t)\right) d x d t
$$

as $\iota \rightarrow \infty$.

Combining the above relation with inequality (21), we have

$$
J\left(u^{*}\right) \leqslant \lim _{t \rightarrow \infty} J\left(u_{l_{L}}\right)
$$

However,

$$
\lim _{l \rightarrow \infty} J\left(u_{l_{l}}\right)=\operatorname{Inf}_{u \in D} J(u)
$$

Thus,

$$
J\left(u^{*}\right)=\operatorname{Inf}_{u \in D} J(u)
$$

and the proof is complete.

REMARK 3.1. Note that Theorem 3.1 contains Theorems 4.1 and 4.2 of Reference 9 as special cases.

In the following theorem, we shall show that the problem $P$ admits a solution without requiring the coefficients and forcing terms of the system dynamic to be 
linear in the control variables if the first term of the cost integrand is the zero-valued function.

THEOREM 3.2. Consider the problem $P$ with $G_{1} \equiv 0$. Let $F_{\kappa}, \hat{F}_{\kappa}$ and $\tilde{F}$ be as defined in equations (6), (7) and (8) respectively. Suppose that the assumptions $(A)$ and the condition (ii) of $\mathscr{G}$ are satisfied and that $\tilde{F}(x, t)$ is convex for each $(x, t) \in \bar{Q}$. Then, if $\tilde{F}$ is a measurable set valued function, the corresponding problem $P$ has a solution.

Proof. Let $\left\{u_{l}\right\}_{l=1}^{\infty} \subset D$ be a sequence so that

$$
\lim _{l \rightarrow \infty} J\left(u_{l}\right)=\operatorname{Inf}_{u \in D} J(u)
$$

From Theorem 2.3, there exists a subsequence $\left\{u_{l}\right\}_{l=1}^{\infty} \subset\left\{u_{l}\right\}_{l=1}^{\infty}$ and a control $u^{*} \in D$ such that

$$
\left.\begin{array}{c}
\phi\left(u_{l_{l}}\right) \rightarrow \phi\left(u^{*}\right), \\
\phi_{x_{i}}\left(u_{l_{l}}\right) \rightarrow \phi_{x_{i}}\left(u^{*}\right), \quad i=1, \ldots, n,
\end{array}\right\} \text { uniformly in } \bar{Q}
$$

as $\imath \rightarrow \infty$. However, the objective functional $J$ of this theorem is identical to $J_{2}$ of Theorem 3.1. Thus, it follows from precisely the same argument as that given for the corresponding part of the proof of Theorem 3.1 that $J\left(u_{l}\right) \rightarrow J\left(u^{*}\right)$ as $\imath \rightarrow \infty$. Since $\left\{u_{l}\right\}_{l=1}^{\infty} \subset D$ is a minimizing sequence, we conclude immediately that $J\left(u^{*}\right)=\operatorname{Inf}_{u \in D} J(u)$. This completes the proof.

\section{Acknowledgements}

The author wishes to acknowledge Dr. S. Nababan and Professor N. U. Ahmed for their valuable discussion and comments. Further, the author also wishes to thank the referees of the paper for their critical comments and valuable suggestions.

\section{References}

[1] N. Dunford and J. T. Schwartz, Linear operators. Part 1. General theory (New York: Interscience, 1958).

[2] H. Hermes and J. P. La Salle, Functional analysis and time optimal control (New York: Academic Press, 1969).

[3] C. J. Himmelberg, M. Q. Jacobs and F. S. Van Vleck, "Measurable multifunctions selectors, and Filippov's implicit functions lemma", J. Math. Anal. Applic. 25 (1969), 276-284.

[4] M. A. Krasnoselskii, Topological methods in the theory of nonlinear integral equations (New York: Macmillan, 1964).

[5] J. L. Lions, Optimal control of systems governed by partial differential equations (New York: Springer-Verlag, 1971). 
[6] A. Plis', "Remark on measurable set-valued functions", Bulletin de l'Académie Polonaise des Sciences, Série des Sciences Math., Astr. et Phy. 9 (1961), 857-859.

[7] F. Riesz and B. Sz.-Nagy, Functional analysis (New York: Frederick Ungar Publishing Co, 1955).

[8] K. L. Teo, "Second order partial differential equations of parabolic type with delayed arguments", Nanta Mathematica 10 (1977), 119-130.

[9] K. L. Teo and N. U. Ahmed, "On the optimal control of a class of systems governed by second order parabolic partial delay-differential equations with first boundary conditions" Annali Mat. di Pura \& Appl. (to appear).

[10] K. L. Teo and N. U. Ahmed, "Optimal feedback control for a class of Stochastic systems", Systems Science 5 (1974), 357-365.

[11] P. K. C. Wong, "Optimal control of parabolic systems with boundary conditions involving time delays", SIAM J. Control 13 (1975), 274-293.

School of Mathematics

University of N.S.W.

P.O. Box 1

Kensington, N.S.W. 2033 